\title{
Époxydation du cyclohexène sur des nouveaux catalyseurs à base de silice mésoporeuse organisée
}

\author{
J. Toufaily ${ }^{1-3}$, T. Hamieh ${ }^{1,4}$, M. Soulard ${ }^{2}$, J.-L. Guth ${ }^{2}$, J. Patarin ${ }^{2}$, \\ G. Baydoun ${ }^{1}$, D. Naoufal ${ }^{1}$, M. Kodeih ${ }^{1}$, M.-B. Fadlallah ${ }^{1}$, \\ I. Zeineddine ${ }^{1}$, M. Elrifai ${ }^{1}$, A. Kanj ${ }^{1}$, A. Houteit ${ }^{1}$ et L. Sierra ${ }^{5}$ \\ ${ }^{1}$ Laboratoire de Chimie Analytique, Matériaux, Surfaces et Interfaces (CHAMSI), \\ Faculté des Sciences, Section 1, Université Libanaise, Hadeth, Beyrouth, Liban \\ ${ }^{2}$ Laboratoire de Matériaux Minéraux, UPRES-A-7016, CNRS, ENSCMu, 3 rue Alfred \\ Werner, 68093 Mulhouse Cedex, France \\ ${ }^{3}$ Laboratoire LACCO - ESIP, 86000 Poitiers, France \\ ${ }^{4}$ Institut de Chimie des Surfaces et Interfaces, ICSI-CNRS, UPR 9096, 15 rue Jean \\ Starcky, BP. 2488, 68057 Mulhouse Cedex, France \\ ${ }^{5}$ Universidad de Antioquia, Medellín, Colombie
}

\begin{abstract}
Heteropoly compounds provide a good basis in acid-base and redox catalysis. In particular, heteropoly acids (HPA) catalyse a wide variety of reactions offering strong options for more efficient and cleaner processes compared to classical inorganic acids. They are more efficient than conventional catalysts, such as silica-alumina, zeolites, etc. Because of their low surface areas $\left(1-10 \mathrm{~m}^{2} / \mathrm{g}\right)$, heteropoly acids are usually supported for catalytic applications. Amorphous silica or all-silica mesoporous molecular sieves are often used as supports for HPA's. These catalysts are usually prepared by the wetness impregnation method. The chief disadvantage of this method is the leaching of the active phase when the catalyst is employed in liquid phase reactions. Our study consisted in the direct incorporation of tungstophosphate species (HPW) into the walls of mesoporous silica. Subsequently, the catalytic activity of a highly-incorporated HPWMCM-41 material was tested in the epoxidation of cyclohexene using $\mathrm{H}_{2} \mathrm{O}_{2}$ as oxidant. On the basis of the experimental results, the samples show a good cyclohexene conversion rate; five main products were detected, i.e. epoxide, cyclohexanediol, 2-cyclohexene-1-ol or 3-cyclohexene-1-ol, cyclohexanone-2hydroxy, and 2-cyclohexene-1,4-diol. The epoxide formed during the reaction seems to undergo further hydrolysis into cyclohexanediol. This could be do to the acidic nature of the catalysts.
\end{abstract}

\section{INTRODUCTION}

Les hétéropolyacides (HPA) de type Keggin, sont utilisés comme catalyseurs acides en raison de leur forte acidité de BrØnsted [1], et aussi comme catalyseurs d'oxydo-réduction [2-3]. Le développement de nouveaux catalyseurs solides présentant les meilleures performances (surface, porosité, sélectivité, etc), a toujours été un défi $[2,4]$. Par ailleurs, un support présentant une structure régulière et une répartition étroite des pores est susceptible de modifier la sélectivité des réactions catalytiques.

Les silices constituent d'excellents supports pour la dispersion des hétéropolyacides [5-7]. Parmi ces silices, les nouvelles phases à mésoporosité contrôlée [8] présentent un très grand intérêt en raison de leur grande surface spécifique $\left(>1000 \mathrm{~m}^{2} \mathrm{~g}^{-1}\right)$ et de la présence de mésopores ayant une 
distribution étroite en diamètre. Récemment, les hétéropolyacides types tungstophosphates ont été introduits par imprégnation soit sur une silice précipitée soit sur une silice mésoporeuse [1].

Dans le présent travail, nous avons préparé et caractérisé de nouveaux catalyseurs solides type tungstophosphate-silice par incorporation du tungstophosphate dans les parois de la silice mésoporeuse au moment de sa formation. Les tungstophosphates précipitant en présence d'ammonium, il n'est pas possible de mettre en cuvre un procédé où la formation de la silice mésoporeuse organisée est obtenue à l'aide $\mathrm{d}^{\prime}$ un seul tensioactif cationique $\mathrm{S}^{+}$type ammonium quaternaire en milieu acide. Une synthèse en milieu basique selon un mécanisme $\mathrm{S}^{+} \mathrm{I}^{-}$est aussi à exclure, car les tungstophosphates sont détruits dans ce milieu. Il reste donc la préparation en milieu légèrement acide, où la polycondensation des acides siliciques peut être contrôlée par un tensioactif non ionique. Dans ce cas, il ne peut pas se produire d'interactions fortes avec les espèces hétéropolyanioniques, aussi un tensioactif cationique $\left(\mathrm{S}^{+}\right)$a été adjoint au non ionique $\left(\mathrm{S}^{0}\right)$. Une étude récente, réalisée au laboratoire [9], sur la synthèse de silice mésoporeuse avec un tensioactif non-ionique a permis de montrer que le $\mathrm{pH}$ de synthèse doit être compris entre 2-3 et 9-10. Compte tenu de l'instabilité des espèces tungstophosphates, le domaine de $\mathrm{pH}$ à été limité entre 3 et 6,5.

Nous avons utilisé principalement l'acide tungstophosphorique $\mathrm{H}_{3} \mathrm{PW}_{12} \mathrm{O}_{40}(\mathrm{PW})$, qui présente l'acidité la plus élevée parmi la série des HPW [3] et examiné successivement le mode opératoire relatif à la synthèse des matériaux, les caractérisations physico-chimiques des phases par microscopie électronique à balayage, diffraction des rayons $\mathrm{X}$, analyses thermiques, adsorptiondésorption d'azote à $77 \mathrm{~K}$ et mesures BET. Malgré de nombreuses questions concernant la nature des phases et la localisation des espèces incorporées, les premières investigations, effectuées dans ce domaine, ont donné des résultats prometteurs. Nous avons ainsi montré, que la synthèse d'une silice mésoporeuse organisée avec incorporation de tungstophosphate peut être réalisée par association de deux sortes de tensioactifs non ionique TX100 et cationique $\mathrm{CTMA}^{+}$. En faisant varier différents paramètres notamment le $\mathrm{pH}$ du milieu, le rapport $\mathrm{S}^{+} / \mathrm{S}^{0}$, la quantité de HPW, on obtient des matériaux caractérisés par une très grande surface spécifique, et contenant l'hétéropolyacide dans ses parois.

\section{EVALUATION CATALYTIQUE DES SOLIDES PREPARES}

Dans cet article, nous avons mis l'accent sur l'étude comparative des propriétés catalytiques des solides mésoporeux obtenus après incorporation des espèces tungstophosphates, lors de la réaction d'époxydation du cyclohexène par l'eau oxygénée. Afin d'obtenir les conditions optimales pour cette réaction, nous avons effectué une série d'expériences en variant les paramètres suivants : le solvant, la température de la réaction, le temps de contact, la masse du catalyseur et le rapport molaire cyclohexène/eau oxygénée. La première série de tests catalytiques a été réalisée dans les mêmes conditions que celles décrites par Zhang et al. [10]. Il s'agit de l'hydroxylation du cyclohexène par $\mathrm{H}_{2} \mathrm{O}_{2}$ en excès, en milieu acide acétique. Des vitesses de réaction assez importantes ont été observées. En effet, après 15 minutes de temps de contact, $99 \%$ du cyclohexène est transformé avec un catalyseur riche en PW, alors que le taux de conversion dans les mêmes conditions, mais en absence de catalyseur n'est que de $1 \%$. Cependant, le milieu acide acétique paraît être "agressif» vis-à-vis du catalyseur, et risque d'extraire des espèces actives. Avec l'isopropanol, les taux de conversion du cyclohexène sont relativement faibles avec une formation peu importante d'époxycyclohexane, malgré une forte consommation en eau oxygénée. Au cours des essais, on s'est aperçu qu'à côté de l'hydroxylation du cyclohexène en présence de $\mathrm{H}_{2} \mathrm{O}_{2}$, une réaction secondaire a lieu: il s'agit de la production de l'acétone par déshydrogénation du 2propanol.

Finalement, l'acétonitrile, solvant polaire et organophile a été testé. Des essais préliminaires ont montré que l'utilisation de ce solvant limitait la perte en $\mathrm{H}_{2} \mathrm{O}_{2}$ observée en milieu isopropanol. Cependant, l'acétonitrile reste le meilleur solvant pour l'oxydation du cyclohexène en présence de $\mathrm{H}_{2} \mathrm{O}_{2}$. Les résultats obtenus en faisant varier les autres paramètres étudiés (température, temps de 
contact, masse du catalyseur et rapport molaire $\mathrm{C}_{6} \mathrm{H}_{10} / \mathrm{H}_{2} \mathrm{O}_{2}$ ) ne sont pas présentés dans cet article, mais nous les avons utilisés pour définir les meilleures conditions opératoires. La composition du mélange réactionnel adoptée après optimisation est celle également utilisées par $\mathrm{Bu}$ et al. [11] : 20 $\mathrm{ml}$ de solvant (acétonitrile); $56 \mathrm{mmol}$ de cyclohexène; $14 \mathrm{mmol}$ d'eau oxygénée à $30 \%$ (soit un rapport molaire $\mathrm{C}_{6} \mathrm{H}_{10} / \mathrm{H}_{2} \mathrm{O}_{2}=4$ ), $200 \mathrm{mg}$ de catalyseur.

Les tests catalytiques ont été réalisés à $70^{\circ} \mathrm{C}$ dans des ballons en verre de $150 \mathrm{ml}$ munis d'un système à reflux, chauffés dans un bain thermostaté avec agitation par un barreau magnétique. Des prélèvements ont été recueillis, puis analysés par chromatographie en phase gazeuse (CPG). L'identification des différents produits a été effectuée à l'aide d'un chromatographe couplé à un spectromètre de masse et également en injectant les produits purs commercialisés. A partir des différents chromatogrammes obtenus, nous avons déterminé les différents pourcentages des produits réactionnels et ceux des produits restants (acétonitrile et cyclohexène). La sélectivité pour chaque produit formé est donnée en \% par rapport à l'ensemble des produits formés alors que le taux de conversion du cyclohexène (en \%) a été calculé en retranchant à 100 le pourcentage du cyclohexène restant. Divers échantillons de catalyseurs ont été étudiés dans cette réaction en faisant varier les différents paramètres.

\section{INFLUENCE DU TAUX DE $\mathrm{H}_{3} \mathrm{PW}_{12} \mathrm{O}_{40}$}

Les échantillons traités dans ce paragraphe, font partie des synthèses avec le système micellaire $\mathrm{TX} 100 / \mathrm{C}_{16} \mathrm{TMABr}$. Dans ce procédé, on engage directement le $\mathrm{H}_{3} \mathrm{PW}_{12} \mathrm{O}_{40}$ dans le milieu réactionnel en présence de(s) tensioactif(s) et du TMOS. Les différents paramètres de ces synthèses ainsi que les principales caractéristiques physico-chimiques des solides sont présentés dans le tableau 1. Dans l'époxydation du cyclohexène en présence de $\mathrm{H}_{2} \mathrm{O}_{2}$, on observe généralement la formation d'une dizaine de produits. Les plus importants sont: l'époxycyclohexane, le cis et/ou trans-1,2-cyclohexanediol, le 2-cyclohexène-1,4-diol et le cyclohexanone-2-hydroxy.

Tableau 1. Paramètres de synthèses dans le système TX100/ $\mathrm{C}_{16} \mathrm{TMABr}$. Composition rapportée à 1 mole de $\mathrm{SiO}_{2}$ est : $\mathrm{mxPW}$, nyTX100, pzC $16 \mathrm{TMABr}$ (composition molaire de référence : $x=0,0043 ; y=0,25 ; z=0,015$ et $m, n$, $p$ sont les facteurs multiplicatifs).

\begin{tabular}{|c|c|c|c|c|c|c|c|}
\hline Echantillon & $\mathbf{P W}_{\mathrm{gel}}$ & TX 100 & $\mathrm{C}_{16} \mathrm{TMABr}$ & $\mathrm{PW} / \mathrm{SiO}_{2}$ solide & $\mathrm{S}_{\mathrm{BET}(\mathrm{m} 2 / \mathrm{g})}$ & $V_{(\mathrm{cm} 3 / \mathrm{g})}$ & $\varnothing_{\mathrm{BJH}(\AA)}$ \\
\hline JOT 038 & 0 & $\mathrm{y}$ & $\mathrm{z}$ & 0 & 1240 & 1 & 26 \\
\hline JOT 002 & $\mathrm{x}$ & $\mathrm{y}$ & $\mathrm{z}$ & 0,00146 & 1400 & 1,05 & 26 \\
\hline JOT 014 & $2,3 x$ & $\mathrm{y}$ & $2 z$ & 0,00467 & 1012 & 0,82 & 25 \\
\hline JOT 017 & $3 x$ & $\mathrm{y}$ & $3 z$ & 0,00820 & 730 & 0,65 & 26 \\
\hline JOT 015 & $4,6 x$ & $\mathrm{y}$ & $4 z$ & 0,01400 & 700 & 0,67 & $26 ; 90$ \\
\hline JOT 016 & $8 x$ & $y$ & $8 z$ & 0,03000 & 375 & 0,46 & 87 \\
\hline
\end{tabular}

Nous avons également identifié d'autres molécules mais à très faibles pourcentages: le cyclohexanol, la cyclohexanone, le 2-cyclohexène-1ol, le 3-cyclohexène-1ol, le 1-cyclohexène-1ol et le 2-cyclohexène-1one.

Les différents essais effectués montrent que le taux de conversion du cyclohexène augmente fortement avec le taux de PW incorporé dans la silice mésoporeuse pour atteindre une valeur limite de $45 \%$ environ. Il semblerait qu'on obtienne une quasi-linéarité entre le taux de conversion en cyclohexène et la teneur en PW incorporé dans le solide. Pour un taux de PW incorporé dans le solide, comparable à celui de PW pur employé en phase homogène, on obtient dans les deux cas une conversion analogue en cyclohexène. Les catalyseurs préparés par incorporation de PW montrent la formation simultanée du cis et trans-1,2-cyclohexanediol avec une sélectivité plus importante pour le trans-1,2-cyclohexanediol dans le cas des matériaux de type MSU. Le cyclohexane-ol, le 3-cyclohexène-1ol et le 2-cyclohexène-1one sont des produits secondaires 
formés en présence de catalyseurs contenant PW incorporé mais inexistants quand PW est introduit par imprégnation ou utilisé en phase homogène.

Lorsque l'ion de Keggin est introduit dans la silice mésoporeuse par imprégnation, on observe une sélectivité importante pour le cis-1,2-cyclohexanediol, impliquant une acidité plus élevée du catalyseur. Lorsque l'espèce de Keggin est utilisée sans support, en phase homogène, on forme uniquement l'isomère cis. Ceci s'explique probablement par la forte lixiviation des espèces actives dans les échantillons imprégnés et dans ce cas, on est en catalyse homogène.

Sur l'ensemble des catalyseurs, on constate une augmentation de la sélectivité en époxyde et une diminution de celle en diols, avec la conversion en cyclohexène. Ce résultat pourrait s'expliquer par une compétition dans les réactions de transformations 1 et 2 . En effet, la succession des réactions est la suivante :

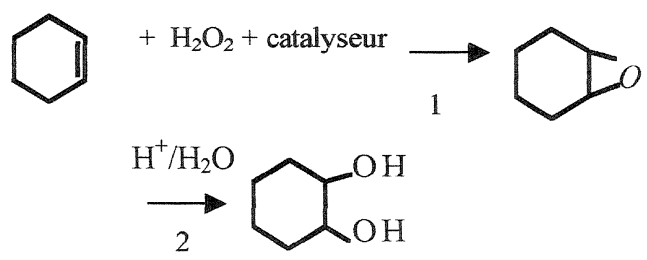

Au début de la réaction, le cyclohexène au contact du catalyseur, se transforme en époxyde, qui en présence des sites acides conduit assez rapidement aux diols. Puis, suite à une désactivation des sites PW, la réaction 2 se trouverait fortement ralenti.

Remerciements. Nous tenons à remercier le comité du Programme franco-libanais CEDRE qui a financé cette étude

\section{Références Bibliographiques}

1. I.V. Kozhevnikov, K.R. Kloetstra, A. Sinnema, H.W. Zandbergen, H. Van Bekkum. J.Mol.Catal.A : Chemicals, 114, 287 (1996).

2. I.V. Kozhevnikov. Chem. Rev. 98, 171 (1998).

3. I.V. Kozhevnikov. Russ. Chem. Rev. 56, 811 (1987).

4. N. Mizuno, M. Misono. Chem. Rev. 98, 199, (1998).

5. D.W. Breck. Zeolite Molecular Sieves, Wiley, New York, (1974).

6. Y. Izumi, K. Urabe and M. Onaka. Zeolite, Clay and Heteropoly Acid in organic Réactions. Kodansha / VCH, Tokyo / Weinheim, (1992).

7. I.V. Kozhevnikov. Catal. Rev. Sci. Eng. 37, 311 (1995).

8. A. Corma. Chem. Rev. 97, 2373 (1997).

9. L. Sierra, J.L. Guth. Microporous and Mesoporous Materials, 27, 243 (1999).

10. Z. Zhang, J. Suo, X. Zhang, S. Li, Chem. Commun., 1998, 241.

11. J. Bu, H.K. Rhee, Studies in Surface Science and Catalysis 129, A. Sayari et al. (Eds.), Elsevier Science B.V., 179 (2000). 